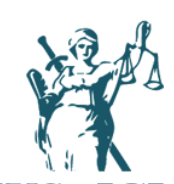

JUSTICIA

ISSN impreso 0124-744

\title{
El uso de la internet versus medios tradicionales de comunicación. Su impacto en la participación política de los jóvenes en el área metropolitana de Monterrey Nuevo León.
}

\author{
The use of the internet versus traditional means of \\ communication. Its impact on the political participation \\ of young people in the metropolitan area of Monterrey \\ Nuevo León.

\section{O uso da internet versus medios tradicionales de comunicación. Su impacto e la participación política de los jóvenes na área metropolitana de Monterrey Nuevo León.}

\author{
Juan de Dios Martínez Villarreal \\ Universidad Autónoma de Nuevo León, México \\ juandediosmtzv@hotmail.com \\ Pedro Paul Rivera Hernández \\ Universidad Autónoma de Nuevo León, México \\ pedro_riverahdz@hotmail.com \\ Karla Eugenia Rodríguez Burgo (iD \\ Universidad Autónoma de Nuevo León, México \\ karoburgos@yahoo.com.mx
}

Recibido: 09 de marzo de 2019 / Aceptado: 4 de julio de 2019

https://doi.org/10.17081/just.24.36.3768

\section{Resumen}

El presente estudio tiene como objetivo principal determinar el nivel explicativo de la comunicación política interactiva, la atención a noticias y programas de política sobre los diferentes tipos de participación política de los jóvenes en el Área Metropolitana de Monterrey, Nuevo León, México. La metodología utilizada fue de corte cuantitativo aplicando una encuesta de opinión. Los resultados más relevantes de la investigación demuestran que la comunicación política interactiva tiene un mayor nivel explicativo sobre la participación política convencional y no convencional en contraste con la atención a noticias.

Palabras Clave: comunicación interactiva; atención a noticias y programas de política; participación política.

Justicia Vol. 24 No. 36: pp. 240-252. Julio - diciembre, 2019. DOI: 10.17081/just.24.36.3768

(c) Copyright 2019 by Martínez Villarreal, J \& e tal.

$$
\text { open } \odot \text { access (c) (i) }
$$




\section{Abstract}

The main purpose of the present study is to determine the explicative level of the politic interactive communication as well as the attention to the news and political programs broadcasted in television about the different ways of youth political participation in the Metropolitan Area of Monterrey, Nuevo León, Mexico. The methodology used was of a quantitative nature using an opinion poll. The most significant outcome shows that the political interactive communication has a higher explicative level of the political conventional and non-conventional participation in contrast to attention to the news.

Keywords: interactive communication; attention to news and political TV shows; political participation

\section{Resumo}

O objetivo principal do presente estudo é identificar o nível explicativo da comunicação interativa política, bem como a atenção às novidades e programas políticos transmitidos na televisão sobre as diferentes formas de participação política da juventude na área metropolitana de Monterrey, Nuevo León, México. A metodologia que foi aplicada foi quantitativa devido à pesquisa conduzida. $O$ resultado mais significativo mostra que a comunicação política interativa tem um nível explicativo mais alto da participação política convencional e não convencional em comparação com o atenção às notícias.

Keywords: comunicação interativa; atenção às notícias e programas políticos; participação política.

Como citar:

Martínez Villarreal, J. de D., Rivera Hernández, P. P., \& Rodríguez Burgos, K. E. (2019). El uso de la internet versus medios tradicionales de comunicación. Su impacto en la participación política de los jóvenes en el área metropolitana de monterrey nuevo león. Justicia, 24(36), 240-252. https://doi.org/10.17081/just.24.36.3768

\section{INTRODUCCIÓN.}

La participación política de los jóvenes es indispensable para la creación de una cultura política democrática; a su vez, es importante mencionar como lo indica AriasCarmona y Alvarado (2015) que los jóvenes son "sujetos políticos activos en la construcción de sus biografías e historias colectivas, que reconocen la diferencia y transforman conscientemente las condiciones -políticas, sociales, económicas y culturales- relacionadas con el ejercicio ciudadano" (p. 599). En este sentido, los jóvenes participan activamente en la toma de las decisiones que los afecten, e inciden en su entorno.

El contexto en el que los jóvenes se han desarrollado en la actualidad ha moldeado su cultura y comportamiento, al estar inmersos en un contexto de desarrollo tecnológico, de problemas de calentamiento global, terrorismo y quiebre de regímenes políticos, a su vez, la Internet (Web. 2) les han permitido una forma distinta de comunicarse con el mundo. (Rodríguez-Burgos, Martínez y Rodríguez-Serpa, 2017) 
En relación a lo anterior, la presente investigación tiene como propósito determinar la influencia de la comunicación política interactiva (uso de internet) y la que se origina en relación a los medios tradicionales de comunicación (televisión y prensa escrita) sobre los diferentes tipos de participación política de los jóvenes en el Área Metropolitana de Monterrey, Nuevo León, México. Contextualizando el estudio se puede indicar que el estado representa el $4.3 \%$ de la población del país; a su vez, el área Metropolitana de Monterrey (AMM) constituye el $87.4 \%$ de la población total del estado de acuerdo al Instituto Nacional de Estadística y Geografía (INEGI). Por otra parte, los jóvenes (18 a 29 años), conforman el $\mathbf{2 8 . 7 5 \%}$ (a marzo de 2019) de la lista nominal que indica el número de ciudadanos con credencial vigente para votar.

Tomando en consideración la representatividad que tienen los jóvenes en el estado, es relevante su participación política para el desarrollo de un sistema democrático, asimismo, es de suma importancia determinar de qué manera la comunicación política es un factor que podría desencadenar en ellos un interés o motivación que se vea reflejado en su intención de participar o influir en la toma de decisiones por parte de la autoridad. Como lo indica Reyes (2007) "la comunicación política en este contexto es el vínculo que facilita el proceso de información en cuanto a su alcance, significado y posibilidades, y es fundamental para el funcionamiento de los sistemas políticos" ( $p$. 114).

El estudio es producto de los resultados de investigación, de corte cuantitativo mediante la aplicación de una encuesta de opinión en el AMM, financiada por el Programa para el Desarrollo Profesional Docente, para el tipo superior (PRODEP). Los objetivos de la investigación son: identificar los niveles de participación política de los jóvenes y el uso de la internet y medios tradicionales para informarse de política; además se busca determinar el nivel explicativo de la atención a noticias, programas de política y la comunicación política interactiva sobre los diferentes tipos de participación política.

En el trabajo se presenta primeramente el marco teórico, donde se definen los conceptos de comunicación política interactiva como un nuevo modelo de comunicación, los medios tradicionales de comunicación y la participación política. Posteriormente se explica la metodología utilizada para la aplicación del instrumento, el análisis de resultados con relación a las variables del estudio, para finalmente presentar la discusión y conclusiones de la investigación.

\section{METOdologíA}

Para la presente investigación se aplicó una encuesta de opinión en el año 2015 representativa de los jóvenes (18 a 29 años) en el AMM, que se conforma por los municipios de Monterrey, Guadalupe, San Nicolás de los Garza, Apodaca, García, Escobedo, San Pedro Garza García, Santa Catarina y Juárez. En lo que respecta al perfil de la muestra se puede indicar que el $52.3 \%$ son mujeres y el $47.7 \%$ son hombres; los ingresos familiares que los encuestados manifiestan indican que el $83 \%$ tiene un ingreso mayor a los $\$ 6000$ pesos mensuales; por otra parte, el $56.2 \%$ son jóvenes de 18 a 21 años y el $43.8 \%$ de 22 a 29 años.

El tamaño de la muestra fue de 1,715 jóvenes aplicando un nivel de confianza del $95 \%$ y un margen de error muestral del $2 \%$. Para medir el nivel de confianza y el margen de 
error,

manejó la fórmula de población infinita, quedando de la siguiente manera:

$n=\left(\left(Z_{\alpha} / 2\right)^{2}(p)(q)\right) / 2$

despejando $n$, resulta:

$E=\left(\left(Z_{\alpha} / 2\right)^{2}(p)(q)\right) / n$

\section{Cuestionario}

Para generar el cuestionario se consideraron escalas de investigaciones previas; es importante comentar que en algunas escalas se efectuaron modificaciones para ajustarlas a las necesidades del estudio.

\section{Variables}

En la creación de la variable atención a noticias y programas de política se preguntó a los jóvenes qué tan frecuente efectuaban actividades como leer noticias en periódicos, ver noticias de televisora y ver programas de sátira o humor político para informarse sobre asuntos de política; tomando como referencia la propuesta de Muñiz (2011) con una escala Likert donde (1) es nada y (5) es bastante.

Para la construcción de la variable se aplicó un análisis factorial dando como resultado un KMO de .717 lo que es importante, la medida de adecuación muestral (MSA) marcó por arriba de 0.6, y las comunalidades estuvieron por encima de 0.5 , por otra parte, la varianza acumulada en un solo factor fue de $82.57 \%$; en el análisis de fiabilidad se alcanzó un Alfa de Cronbach $(\alpha)$ de .880; estos resultados le da validez y fiabilidad a los ítems que conformaron la variable.

Por otra parte, para crear la variable comunicación política interactiva se preguntó a los encuestados con qué frecuencia utilizaban Internet para realizar diferentes actividades con un propósito político; tomándose la propuesta de Shah et al. (2007) con una escala Likert donde (1) es nada y (5) es bastante. En la construcción de la variable se llevó a cabo un análisis factorial dando como resultado un $K M O$ de .813 lo que es significativo, la medida de adecuación muestral (MSA) marcó por arriba de 0.6, y las comunalidades marcaron por encima de 0.5 ; la varianza acumulada en un solo factor fue de $73.58 \%$; en el análisis de fiabilidad se obtuvo un Alfa de Cronbach $(\alpha)$ de .901; lo anterior le da validez y fiabilidad.

En lo que corresponde a la variable de participación política se dividió en tres tipos, siendo la primera la participación política electoral tomando la propuesta del ENCUP 2012, se preguntó de manera directa a los encuestados su participación en las elecciones para gobernador del estado, manejando una escala dicotómica donde (0) es no y (1) es sí.

A su vez, para la creación de la variable participación política convencional se toma como referencia la ENCUP (2012) manejando una escala de Likert de 5 puntos donde (1) es nada y (5) es bastante; se preguntó a los jóvenes si había intentado convencer a sus 
amigos para que voten o no por un candidato, asistido a reuniones de partidos políticos, trabajar para algún candidato o partido político, etc.; para generar el constructo se aplicó un análisis factorial dando como resultado un KMO de .865 lo cual fue relevante, la medida de adecuación muestral (MSA) estuvo por encima de 0.6 , las comunalidades fueron arriba de 0.5 ; la varianza acumulada en un solo factor fue de $68.18 \%$; en relación al análisis de fiabilidad se alcanzó un Alfa de Cronbach $(\alpha)$ de .878; estos indicadores le dan fiabilidad y validez a los ítems.

Para la creación de la variable participación política no convencional se toma como referencia la ENCUP (2012) aplicando una escala de Likert de 5 puntos donde (1) es nada y (5) es bastante, preguntando a los jóvenes su participación en acciones como plantones, colocación de mantas o bloqueo de calles, en marchas o boicot de actos públicos o han hecho solicitudes o firmado documentos en señal de protesta o solicitando algo; a su vez, se realizó un análisis factorial dando como resultado un KMO de .852 lo cual fue significativo, la medida de adecuación muestral (MSA) marcaron por encima de 0.6, las comunalidades estuvieron por encima de 0.5; la varianza acumulada en un solo factor fue de $61.49 \%$; en el análisis de fiabilidad se obtuvo un Alfa de Cronbach $(\alpha)$ de .842; estos resultados le dan fiabilidad y validez a los ítems que forman la variable.

Tabla 1. Análisis factorial y de fiabilidad

\begin{tabular}{cccc}
\hline Variables o constructos & KMO & $\begin{array}{c}\text { Alfa de } \\
\text { Cronbach } \alpha\end{array}$ & $\begin{array}{c}\text { Varianza } \\
\text { acumulada }\end{array}$ \\
\hline $\begin{array}{c}\text { Atención a noticias y a programas de } \\
\text { política }\end{array}$ & .717 & .880 & $82.57 \%$ \\
\hline $\begin{array}{c}\text { Comunicación interactiva } \\
\text { Participación política convencional }\end{array}$ & .813 & .901 & $73.58 \%$ \\
\hline $\begin{array}{c}\text { Participación política no } \\
\text { convencional }\end{array}$ & .852 & .878 & $68.18 \%$ \\
\hline
\end{tabular}

Fuente: Elaboración propia

\section{RESULTADOS}

En lo que respecta a la variable atención a noticias y programas de política $(M=2.32$, $\mathrm{DE}=.773$ ) como constructo es baja, ya que se encuentra por debajo de la media aritmética; la actividad con mayor incidencia por parte de los jóvenes es ver noticias de televisoras ( $M=2.28, D E=1.09)$; en un segundo término se puede encontrar a la lectura de noticias en periódicos ( $M=2.28, D E=1.09)$; a su vez, los resultados más bajos se observan sobre ver programas de sátira o humor ( $\mathrm{M}=1.90, \mathrm{DE}=1.14)$.

En relación a la variable comunicación política interactiva $(M=1.91, D E=.857)$ en general (constructo) es bastante baja, por debajo de la media promedio; asimismo, las actividades que realizan los jóvenes con mayor frecuencia son compartir noticias, videos o posts sobre política en internet $(M=2.32, D E=1.19)$ e intercambiar opiniones sobre política por correo electrónico, redes sociales, sitios web u otras aplicaciones de internet ( $M=2.27, D E=1.28)$; por su parte, los resultados de las demás actividades como participar en discusiones políticas a través de la red $(M=1.77, D E=1.09)$, subir a internet videos, animaciones, audios fotos, para expresar su postura política $(M=1.68, D E=1.02)$ y escribir 
sobre asuntos políticos en blogs ( $M=1.52, D E=.905)$ son las actividades que con menos frecuencia llevan a cabo los jóvenes.

En la participación política electoral se reportó que el 85.3\% de los jóvenes votó en las elecciones para gobernador, por otra parte, la variable se transformó en escala de Likert para estar acorde a las demás variables, en este sentido, como ya se observó la participación electoral es alta ( $\mathrm{M}=4.44, \mathrm{DE}=1.38)$.

La participación política convencional de los jóvenes como constructo es muy baja ( $M=1.71, D E=.747)$; la mayor participación se observa al intentar convencer a sus amigos para que voten por los candidatos que ellos cree que tienen que ganar una elección ( $\mathrm{M}=1.93, \mathrm{DE}=1.13$ ); a su vez, la menor participación se identifica al cuestionarlos sobre su trabajo para algún candidato o partido político (M=1.58, DE=.984).

Asimismo, en relación a la participación política no convencional como variable es aún más baja que la convencional ( $\mathrm{M}=1.54, \mathrm{DE}=.709)$, la mayor participación se observa al firmar documentos en señal de protesta o solicitando algo ( $M=1.83, \mathrm{DE}=1.10)$; por otra parte, la actividad que los encuestados realizan con menor frecuencia es la participación en marchas o boicot de actos públicos al no estar de acuerdo con alguna decisión del gobierno ( $\mathrm{M}=1.83, \mathrm{DE}=1.10)$.

Tabla 2. Resultados descriptivos de las variables del estudio

\begin{tabular}{ccc}
\hline Variables o constructos & Media & Desviación estándar \\
\hline $\begin{array}{c}\text { Atención a noticias y a programas } \\
\text { de política }\end{array}$ & 2.32 & .773 \\
\hline Comunicación interactiva & 1.91 & .857 \\
\hline Participación política electoral & 4.44 & 1.38 \\
\hline Participación política convencional & 1.71 & .747 \\
\hline $\begin{array}{c}\text { Participación política no } \\
\text { convencional }\end{array}$ & 1.54 & .709 \\
\hline
\end{tabular}

Fuente: Elaboración propia

Nota: $N=1715$ casos. Los resultados fueron estadísticamente significativos a un nivel de confianza de ${ }^{*} p<.05 ;{ }^{* *} p<.01 ;{ }^{* *} p<.001$

Al finalizar los análisis descriptivos se analizó la relación o covarianza existente entre las variables participación política electoral, convencional y no convencional con la atención a noticias y programas de política donde se reporta que la relación entre la atención a noticias y programas de política con la participación política electoral es positiva $(r=.076, p<.01)$, a su vez, la relación con la participación política convencional es significativa $(r=.226, p<.01)$, finalmente la relación con la participación política no convencional también es positiva $(r=.226, p<.01)$.

Tabla 3. Correlaciones entre participación electoral, participación política convencional, participación política no convencional y atención a noticias y programas de política.

\begin{tabular}{llllll}
\hline Variables & 1 & 2 & 3 & 4 \\
\hline
\end{tabular}




\begin{tabular}{cccc}
\hline Participación electoral & $-050^{*}$ & .012 & $.076^{* *}$ \\
\hline $\begin{array}{c}\text { Participación política } \\
\text { convencional }\end{array}$ & - & $.0541^{* *}$ & $.226^{* *}$ \\
\hline $\begin{array}{c}\text { Participación política no } \\
\text { convencional }\end{array}$ & - & $.172^{*}$ \\
\hline $\begin{array}{c}\text { Atención a noticias y programas } \\
\text { de política }\end{array}$ & & & - \\
\hline
\end{tabular}

Fuente: Elaboración propia

Nota: $N=1715$ casos. Los resultados fueron estadísticamente significativos a un nivel de confianza de ${ }^{*} p<.05 ;{ }^{* *} p<.01 ;{ }^{* *} p<.001$

Por otra parte, se buscó la relación o covarianza existente entre las variables participación política electoral, convencional, no convencional y la comunicación política interactiva donde se obtienen los siguientes resultados: la relación entre la comunicación política interactiva con la participación política electoral es positiva ( $r=$ $.067, \mathrm{p}<.01)$, asimismo, su relación con la participación política convencional es significativa $(r=.431, p<.01)$, la relación con la participación política no convencional también es importante $(r=.402, p<.01)$.

Tabla 4. Correlaciones entre participación electoral, participación política convencional, participación política no convencional y comunicación política interactiva.

\begin{tabular}{|c|c|c|c|c|}
\hline Variables & 1 & 2 & 3 & 4 \\
\hline Participación electoral & - & $.050^{*}$ & .012 & $.067^{* *}$ \\
\hline $\begin{array}{l}\text { Participación política } \\
\text { convencional }\end{array}$ & & - & $.0541^{* *}$ & $.431 * *$ \\
\hline $\begin{array}{c}\text { Participación política no } \\
\text { convencional }\end{array}$ & & & - & $.402 *$ \\
\hline $\begin{array}{c}\text { Comunicación política } \\
\text { interactiva }\end{array}$ & & & & - \\
\hline
\end{tabular}

Fuente: Elaboración propia

Nota: $\mathrm{N}=1715$ casos. Los resultados fueron estadísticamente significativos a un nivel de confianza de $* p<.05 ; * * p<.01 ; * * * p<.001$

Como última etapa de análisis con el objetivo de probar las hipótesis de investigación se llevó a cabo una regresión lineal simple para determinar el nivel explicativo de las variables atención a noticias y programas de política y comunicación política interactiva sobre los diferentes tipos de participación política que mostraron asociación de acuerdo a la prueba $r$ de Pearson. 
Primeramente, se buscó determinar el nivel explicativo de la variable atención a noticias y programas de política sobre las variables independientes. En lo que corresponde a la participación electoral la variable independiente explica el $0.5 \%$ de la varianza $\left(R^{\wedge} 2=.005\right)$, por otra parte, la variable independiente cumple con el supuesto de independencia respecto a la dependiente, ya que el valor del test Durbin - Watson es de $D=1.75$, por lo que está dentro de los parámetros reconocidos. La atención a noticias y programas de política (con un valor de $\beta=0,076 ; p<.01$ ), no tienen un nivel explicativo significativo sobre la participación electoral.

Asimismo, en relación a la participación política convencional la variable independiente explica el $5.1 \%$ de la varianza $\left(R^{\wedge} 2=.051\right)$, a su vez, la variable independiente cumple con el supuesto de independencia respecto a la dependiente, ya que el valor del test Durbin - Watson es de $D=1.72$, por lo que está dentro de los parámetros normales. La atención a noticias y programas de política (con un valor de $\beta$ $=0,226 ; p<.001$ ), explica positivamente la participación política convencional, es decir, niveles altos de atención a noticias y programas de política, generarán mayor participación política convencional.

Por otra parte, en lo que corresponde a la participación política no convencional la variable independiente explica el $2.9 \%$ de la varianza $\left(R^{\wedge} 2=.029\right)$, cabe mencionar que la variable independiente cumple con el supuesto de independencia respecto a la dependiente, ya que el valor del test Durbin - Watson es de $D=1.70$, por lo que está dentro del parámetro estándar. La atención a noticias y programas de política (con un valor de $\beta=0,172 ; p<.001$ ), explica de manera positiva la participación política no convencional, es decir, una mayor atención a noticias y programas de política, provocará una mayor participación política no convencional.

Tabla 5. Nivel explicativo de la atención a noticias y programas de política sobre las variables dependientes.

\begin{tabular}{|c|c|c|c|}
\hline Variable & $\begin{array}{c}\text { Modelo } 1 \\
\beta \\
\text { Participación } \\
\text { electoral }\end{array}$ & $\begin{array}{c}\text { Modelo } 2 \\
\beta \\
\text { Participación } \\
\text { política } \\
\text { convencional }\end{array}$ & $\begin{array}{c}\text { Modelo } 3 \\
\beta \\
\text { Participación } \\
\text { política no } \\
\text { convencional }\end{array}$ \\
\hline $\begin{array}{l}\text { Atención a noticias y } \\
\text { programas de política }\end{array}$ & $.076^{*}$ & $226 * * *$ & $.172 * * *$ \\
\hline$R^{2}$ & .005 & .051 & .029 \\
\hline
\end{tabular}

Fuente: Elaboración propia

En el segundo término se trató de identificar el nivel explicativo de la variable comunicación política interactiva sobre las variables independientes. En relación a la participación electoral la variable independiente explica el $0.4 \%$ de la varianza $\left(R^{\wedge} 2=\right.$ .004), la variable independiente cumple con el supuesto de independencia respecto a la dependiente, ya que el valor del test Durbin - Watson es de $D=1.75$, por lo que está dentro de los parámetros normales. La comunicación política interactiva (con un valor de $\beta=0,067 ; p<.01)$, prácticamente no explica la participación electoral.

En relación a la participación política convencional la variable independiente explica el $18.6 \%$ de la varianza $\left(R^{\wedge} 2=.186\right)$, la variable independiente cumple con el supuesto de 
independencia respecto a la dependiente, ya que el valor del test Durbin - Watson es de $D=1.75$, por lo que está dentro de los parámetros permitidos. La comunicación política interactiva (con un valor de $\beta=0,431 ; p<.001$ ), explica de manera significativa la participación política convencional, es decir, una mayor comunicación política interactiva, generarán mayor participación política convencional.

En lo que corresponde a la participación política no convencional la variable independiente explica el $16.1 \%$ de la varianza $\left(R^{\wedge} 2=.161\right)$, la variable independiente cumple con el supuesto de independencia respecto a la dependiente, ya que el valor del test Durbin - Watson es de $D=1.72$, por lo que está dentro del parámetro normal. La comunicación política interactiva (con un valor de $\beta=0,402 ; p<.001$ ), explica de manera significativa la participación política no convencional, es decir, niveles altos de comunicación política interactiva, incrementarán la participación política no convencional.

Tabla 6. Nivel explicativo de la comunicación política interactiva sobre las variables dependientes.

\begin{tabular}{|c|c|c|c|}
\hline Variable & $\begin{array}{c}\text { Modelo } 1 \\
\beta \\
\text { Participación } \\
\text { electoral }\end{array}$ & $\begin{array}{c}\text { Modelo } 2 \\
\beta \\
\text { Participación } \\
\text { política } \\
\text { convencional }\end{array}$ & $\begin{array}{c}\text { Modelo } 3 \\
\beta \\
\text { Participación } \\
\text { política no } \\
\text { convencional }\end{array}$ \\
\hline $\begin{array}{c}\text { Comunicación } \\
\text { política interactiva }\end{array}$ & $.067^{* *}$ & $431^{* * *}$ & $.402 * * *$ \\
\hline$R^{2}$ & .004 & .186 & .161 \\
\hline
\end{tabular}

Fuente: Elaboración propia

\section{DISCUSIÓN Y CONCLUSIONES}

Los jóvenes representan un potencial importante en el desarrollo de un sistema democrático y la comunicación política es un factor determinante para que ellos participen en materia política. De ahí la importancia de los resultados obtenidos en el estudio que nos permiten una discusión amplia sobre la injerencia de la comunicación política tanto interactiva (uso de internet) como tradicional (prensa escrita y televisión) en relación a los diferentes tipos de participación política.

Para dar respuesta a las preguntas de investigación se utilizaron los resultados descriptivos del estudio, con respecto a la primera pregunta de investigación se puede indicar que la participación política electoral es alta, inclusive por encima de la participación a nivel federal y del estado, en contra parte, la participación política convencional y no convencional es bastante baja, en este sentido, los jóvenes presentan una apatía y desafección política en relación a este tipo de actividades, resultados semejantes a los presentado en otros estudios (Brussino et al., 2009; Díaz \& Carmona, 2013; Duarte \& Jaramillo, 2009; Gutiérrez, 2011; \& Martínez \& Alamilla, 2015); y revalidan lo establecido en la ENCUP (2012) donde se menciona que ocho de cada diez ciudadanos está de acuerdo o muy de acuerdo en que el ejercicio del voto es el único 
mecanismo con el que se cuentan para expresar si el gobierno hace bien o mal las cosas; asimismo, la encuesta nacional de cultura política de los jóvenes 2012 indica que los jóvenes en México consideran útil votar ya que sirve para que mejoren las condiciones de vida, para que haya más justicia social y para exigir a los políticos el cumplimiento de promesas (Gómez, Tejera \& Aguilar 2103).

En relación a lo anterior, se podría pensar que los jóvenes consideran a la participación electoral prácticamente como la única manera de influir en la toma de decisiones de sus gobernantes, en este sentido, la participación electoral es la práctica más utilizada para participar en política (Grossi, Herrero, Rodríguez \& Fernández 2000; Sabucedo \& Arce 1991; Somuano 2005; Martínez \& Wright). Por otra parte, el Informe País sobre la Calidad de la Ciudadanía en México (2015) expone que las personas que participaron en actividades no electorales no obtuvieron los resultados deseados, por lo que esto podría ser un factor que influya en la disminución de incentivos para participar en este tipo de actividades.

En lo que respecta, a la segunda pregunta de investigación en relación al tiempo que dedican los jóvenes a la atención a noticias y programas de política se reporta que es bajo, aunque cabe mencionar que es la televisión el medio que más utilizan los jóvenes para informarse de política, estos resultados son semejantes a los presentados por la ENCUP (2012) donde se indica que el medio que más utilizan los mexicanos para informarse de lo que pasa en materia política es la televisión; a su vez, el trabajo llevado a cabo en Nuevo León por Cantú, Flores, Gutiérrez, Panszi y Yarto (2009) revela que los medios de comunicación que tienen una mayor influencia en el elector del estado son los noticieros y la televisión. Al contrastar el resultado de la investigación con los estudios mencionados se podría indicar que, aunque la televisión podría ser el medio más utilizado para informarse de política, no es necesariamente a este tipo de programas a los que se le dedica mayor tiempo.

Los jóvenes en México tienen un consumo alto de Internet como lo indica la Encuesta Nacional sobre Disponibilidad y Uso de Tecnologías de la Información en los Hogares, 2017 (ENDUTIH) realizada por el INEGI, donde se muestra que el 83.7\% de los jóvenes de 18 a 34 años utilizan este medio para llevar a cabo diferentes tipos de actividades; pero en lo que corresponde al uso de éste en materia política en relación a los resultados del estudio es bastante bajo, dentro de las actividades que realizan con mayor frecuencia se encuentran compartir noticias, videos o spots sobre política, en este sentido, y en relación a lo reportado por la investigación se podría revelar que la actividad en la Internet de los jóvenes en materia política es limitada.

En lo que corresponde a la primera hipótesis de investigación se puede indicar que el nivel explicativo de la variable atención a noticias y programas de política sobre los diferentes tipos de participación política es bajo, el nivel explicativo sobre la participación política electoral es prácticamente nulo, por otra parte, en relación a la participación política convencional y no convencional es moderado; como ya se mencionó y lo confirman la ENCUP (2012), la televisión es el medio de comunicación más utilizado por los jóvenes, más esto no se refleja en el incremento de su participación política. En relación a lo anterior, se puede mencionar que, aunque se cumple tanto con la hipótesis general como con las particulares el nivel explicativo no es relevante.

Justicia Vol. 24 No. 36: pp. 240-252. julio - diciembre, 2019. DOI: 10.17081/just.24.36.3768 
Es importante destacar que los resultados de la investigación realizada por Martínez y Maldonado (2017) al hacer un estudio comparativo de los años 2013 y 2015 en el AMM en jóvenes de 18 a 29 años en relación al impacto de la atención a noticias y programas de política se muestra que el nivel explicativo que esta variable tiene sobre la participación política convencional y no convencional disminuye drásticamente de un estudio a otro. Asimismo, se puede comentar que los resultados del presente trabajo en relación a la variable atención a noticias y programas de política se aproximan a las teorías de la movilización política, más no de manera contundente ya que como se menciona en el contexto estudiado los medios tradicionales de comunicación a través del tiempo han disminuido notablemente su influencia en los diferentes tipos de participación política de los jóvenes.

Por otra parte, el nivel explicativo de la comunicación política interactiva frente a la participación electoral no es relevante, se podría decir, que este tipo de comunicación no promueve en los ciudadanos la intención de votar en una elección; en contra parte, el nivel explicativo de esta variable sobre la participación política convencional y no convencional es importante; estos resultados nos indican que el uso de las TICs están generando una nueva cultura digital que se ve reflejada en los procesos políticos, en este sentido, se puede afirmar que el uso de la red como actividad interactiva puede influir de manera relevante en la participación política (Campos, 2008 \& Pérez, Peña, Genaut, Iturregui \& Mendiguren, 2013).

Se puede concluir que al comparar atención a noticias y programas de política (medios tradicionales) con la comunicación política interactiva (uso de la internet) esta última tiene un mayor nivel explicativo sobre participación política convencional y no convencional, parecería que la nueva forma de comunicación interactiva entre los jóvenes esta ganado cada día más adeptos para promover aquellas acciones políticas que quedarían fuera de la participación electoral, es decir, este tipo de comunicación fomenta actividades tanto individuales como colectivas que le permiten a los ciudadanos incidir en la toma de decisiones de sus gobernantes. En lo que corresponde, a las teorías que buscan explicar la correlación entre la comunicación política con algunas actitudes políticas del ciudadano, los resultados del presente trabajo en relación a la participación convencional y no convencional se inclinan a las teorías relacionadas con la movilización política, aunque como ya se mencionó en lo que corresponde a la atención a noticias y programas de política no es concluyente, a diferencia de la comunicación política interactiva donde se observa de manera más clara esta relación.

En lo que corresponde, a la participación electoral ni la atención noticias y programas de política y la comunicación política interactiva tienen un nivel explicativo relevante, en este sentido, se puede indicar que el voto de los jóvenes no depende de manera importante de estos factores, aunque sería importante determinar si estos influyen en la decisión, opinión y actitudes que tienen los ciudadanos frente a los candidatos.

\section{REFERENCIAS}

Arias-Cardona, A. M. \& Alvarado, S. V. (2015). Jóvenes y política: de la participación formal a la movilización informal. [Youth and Politics: from formal participation

Justicia Vol. 24 No. 36: pp. 240-252. julio - diciembre, 2019. DOI: 10.17081/just.24.36.3768 
to informal mobilization]. Revista Latinoamericana de Ciencias Sociales, Niñez y Juventud, 13(2), 581-594.

Brussino, S., Rabbia, H. H., \& Sorribas, P. (2009). Perfiles sociocognitivos de la participación política de los jóvenes. [Sociocognitive profiles of the political participation of youth]. Interamerican Journal of Psychology, 43(2), 279-287.

Campos Freire, F. (2008). Las redes sociales trastocan los modelos de los medios de comunicación tradicionales. [Social networks alter the models of traditional media]. Revista Latina de Comunicación Social. 63, 1-9.

Cantú Escalante, J., Flores Rodríguez, P., Gutiérrez Garza, E.M., Panszi Artezán, S., Yarto Wong, M. (2009). Perfil del Elector Neoleonés. [Neoleonés Elector Profile]. En Comisión Estatal Electoral Nuevo León y Universidad Autónoma de Nuevo León (Eds). Perfil del Elector Neolonés (65-90). México.

Díaz, A. \& Carmona, O. L. (2013). Rasgos del sujeto político en jóvenes universitarios. [Political subject features in university youths]. Revista Tesis Psicológica, 8(2), 164-177.

Duarte Moller A. \& Jaramillo Cardona M.C. (2009). Cultura política, participación ciudadana y consolidación democrática en México. [Political culture, citizen participation and democratic consolidation in Mexico]. Espiral, Estudios sobre Estado y Sociedad, 16(46), 137-171.

Encuesta Nacional sobre Cultura Política y Prácticas Ciudadanas (ENCUP). (2012). Resultados de la Encuesta Nacional sobre Cultura Política y Prácticas Ciudadanas. [Results from National Survey on Political Culture and Citizen Practices]. México, F.D.: Secretaría de Gobernación.

Gómez Tagle, Silvia; Tejera Gaona, Héctor \& Jesús Aguilar López (2013). Informe de la encuesta "La cultura política de los jóvenes en México" para el Instituto Federal Electoral. [Report of the survey "the political culture of young people in Mexico" for the Federal Electoral Institute], México: El Colegio de México.

Grossi, F. J., Herrero, F. J., Rodríguez, F. J., \& Fernández Alonso, J. A. (2000). Conducta de voto en los jóvenes. [Voting behavior in youth people]. Psicothema, 12(2), 255259. 'ín:

Gutiérrez L., R. (2011). Los déficits de ciudadanía en el proceso de democratización en México. [Citizenship deficits in the process of democratization in Mexico]. In J. Flores Dávila, A 50 años de la cultura cívica: Pensamientos y reflexiones en honor al profesor Sidney Verba (pp. 111- 117). México: Instituto de Investigaciones Jurídicas. UNAM.

INE (2015) Informe país sobre la calidad de la ciudadanía. [Country report in the quality of citizenship). [2 a reimpr.] [pdf]. México: Instituto Nacional Electoral. Disponible

en 
$<$ http://www.ine.mx/archivos2/s/deceyec/EducacionCivica/Documento_Princi pal_23Nov.pdf>.

INEGI (2017) Encuesta Nacional sobre Disponibilidad y uso de tecnologías de la información en los hogares (ENDUTIH). [The National Survey on Availability and Use of Information Technologies in Households]. México: Instituto Nacional de Estadística y Geografía. Disponible en http://www.beta.inegi.org.mx/contenidos/saladeprensa/boletines/2018/OtrT emEcon/ENDUTIH2018_02.pdf

Martínez Villarreal, J.D. \& Alamilla Sosa, J.M. (2015). La participación política de los jóvenes en el Área Metropolitana de Monterrey Nuevo León. Un enfoque sistémico. [The political participation of young people in the Metropolitan Area of Monterrey Nuevo León. A systemic approach]. Política, globalidad y Ciudadanía. 1(2), 59-76.

Martínez Villarreal, J.D. \& Wright, C. (2016). Participación política y actitudes ciudadanas en Nuevo León: Un análisis del escenario pre-electoral 2015. [Political participation and citizen attitudes in Nuevo León: An analysis of the pre-election scenario 2015]. In G. Tamez González, \& X. Arango Morales, Panorama de la Reforma Electoral 2014 en México Debates y Análisis con Experiencia en el Estado de Nuevo León (pp. 69-87) Ciudad de México: Tirant Lo Blanch.

Martínez, J. D. \& Maldonado, L. (2017). El efecto de la atención a noticias en la participación política de los jóvenes en el Área Metropolitana de Monterrey, México. Un estudio longitudinal. [The effect of attention to news on the political participation of young people in the Metropolitan Area of Monterrey, Mexico. A longitudinal study]. Cuadernos.info. (40), 39-52. https://doi.org/10.7764/cdi.40.1095.

Muñiz, C., Maldonado, L. y López, R.E. (mayo 2011). Efectos de las prácticas comunicativas sobre las actitudes políticas de los jóvenes. El caso Monterrey, México. [Effects of communicative practices on the political attitudes of young people. The Monterrey case, Mexico]. Comunicación presentada en el IV Congreso Latinoamericano de Opinión Pública de WAPOR, Belo Horizonte, Brasil.

Pérez, J.A.; Peña, S.; Genaut, A.; Iturregui, L.; \& Mendiguren, T. (2013). Comunicación política e Internet: Estrategias online de los partidos políticos vascos en las elecciones autonómicas de 2009. [Political communications and the Internet: Basque political parties' on-line strategies in 2009 regional elections]. Mediatika. 14, 125-150.

Reyes Montes, M. C. (2007). Comunicación política y medios en México: el caso de la reforma a la Ley Federal de Radio y televisión. [Political communication and media in Mexico: The case of the reform to the Federal Law of Radio and Television]. Convergencia. Revista de Ciencias Sociales. 14(43), 105-136.

Rodríguez-Burgos, K., Martínez Cárdenas, A. A., \& Rodríguez-Serpa, F. A. (2017). Estudio empírico sobre los valores democráticos de tolerancia y respeto en la 
generación milenaria. [Empirical study about the democratic values of tolerance and respect in the millennial generation]. Justicia, (31), 135-150. http://dx.doi.org/ 10.17081/just.22.31.2603

Sabucedo, J. M., \& Arce C. (1991). Types of political participation: A multidimensional analysis. Europea Journal of Political Research. 20(1), 93-102. https://doi.org/10.1111/j.1475-6765.1991.tb00257.x.

Shah, D. V., Cho, J., Nah, S., Gotlieb, M. R., Hwang, H., Leem, N., Scholl, R. M. \& McLeod, D. M. (2007). Campaign Ads, Online Messaging, and Participation: Extending the Communication Mediation Model. Journal of Communication, 57(4), 676-703.

Somuano Ventura, M. F. (2005). Más allá del voto: Modos de participación política no electoral en México. [Beyond the vote: ways of non-electoral political participation in Mexico]. Foro Internacional, 45(1), 65-88. 